Received: $\quad 2015.09 .25$

Accepted: 2015.11.05

Published: 2016.05 .15

\title{
Anti-PLA ${ }_{2}$ Antibodies in Chinese Patients with Membranous Nephropathy
}

Authors' Contribution: Study Design A Data Collection B Statistical Analysis C Data Interpretation D Manuscript Preparation E Literature Search F Funds Collection G

\author{
CEF 1 Xin Li* \\ BF 1 Dong Wei* \\ BF 2 Zhanmei Zhou \\ BD 2 Baoguo Wang \\ DF 3 Ya Xu \\ CE 1 Jie Pan \\ BF 1 Chunli Yang \\ CF 1 Jie Lu \\ ADG 1 Yurong Qiu
}

1 Division of Clinical Laboratory, Nangfang Hospital, Southern Medical University, Guangzhou, Guangdong, P.R. China

2 Division of Nephrology, Nanfang Hospital, Southern Medical University, Guangdong Provincial Institute of Nephrology, Guangzhou, Guangdong, P.R. China 3 EUROIMmUN Medical Diagnostics (China) Co., Ltd., Beijing, P.R. China
Corresponding Author: Source of support:

* Co-first author

Yurong Qiu, e-mail: qiuyuronggz@126.com

This work was supported by the Key Project of the Department of Education of Guangdong Province (No. 2012KJCX0029) and Enterprise-University-Institute Binding Project of Department of Education of Guangdong Province (No. 2012B091100461)

Background: This study used 2 standardized methods to evaluate anti-PLA 2 antibody in sera of Chinese patients with primary membranous nephropathy (PMN) and to determine whether immunological reactivity reflected by antibody titer correlates with kidney function parameters.

Material/Methods: Overall, 82 subjects with biopsy-proven primary membranous nephropathy (PMN), 22 cases with secondary membranous nephropathy (SMN), 40 non-MN patients with established glomerulonephritis, and 20 healthy volunteers were recruited from the Division of Nephrology, Nanfang Hospital, China. Anti-PLA $R$ antibody in the serum of each patient was evaluated by both recombinant cell-based indirect immunofluorescence assay (RC-IFA) and enzyme-linked immunosorbent assay (ELISA). Kidney function was assessed by proteinuria for 24 hours, serum albumin, blood urea nitrogen (BUN), serum creatine, and serum cystatin C. We assessed the correlation between anti-PLA 2 R antibody levels and clinical parameters in the PMN patients.

Results: $\quad$ Fifty-three patients with PMN (64.6\%) were positive for anti-PLA $R$ antibody. The level of antibody determined by RC-IFA ranged from 1: 10 to 1: 1000 and 0 to $1423 \mathrm{RU} / \mathrm{ml}$ by ELISA. The 2 anti-PLA $\mathrm{R}$ test systems correlated very well with each other and reached an agreement of $95.7 \%$ for PMN patients. The level of antibody detected by ELISA in patients with PMN was also significantly correlated with proteinuria and nephritic-range proteinuria (>3.5 g/day).

Conclusions: $\quad$ Anti-PLA $\mathrm{R}_{2}$ antibody is sensitive and extremely specific for diagnosis of Chinese patients with primary membranous nephropathy. Concentration of autoantibody against PLA $R$ may be an ideal marker for monitoring the activity of immunological disease.

MeSH Keywords:

Autoantibodies • Glomerulonephritis, Membranous • Receptors, Phospholipase A2

Full-text PDF: $\quad$ http://www.medscimonit.com/abstract/index/idArt/896090

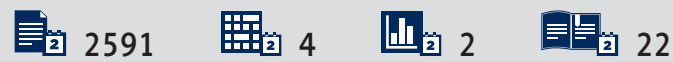




\section{Background}

Primary membranous nephropathy (PMN) is a common cause of nephritic syndrome in adults. Although PMN has been known as an immunological disease for more than 50 years, Beck et al. [1] first identified $M$-type phospholipase $A_{2}$ receptor $\left(P L A_{2} R\right)$ as the major antigen in 2009. PLA $R$ is a transmembrane receptor that presents on human glomerular podocytes. It has been demonstrated that anti-PLA $R$ antibodies are present in kidney eluent and colocalize with PLA $R$ in glomeruli [1]. In most patients affected by PMN, the disease process initiates upon binding of circulating autoantibodies to $P L A_{2} R$ on podocytes. Subsequent sub-epithelial immune deposits and complement activation lead to damage of the filtration barrier and cause proteinuria [2,3]. The pathogenic role of $P L A_{2} R$ is also supported by the strong association between the PLA $R$ gene variant and PMN [4].

In this setting, subsequent research has focused on the clinical utility of anti-PLA $\mathrm{R}_{2}$ antibodies. Several studies have shown that the prevalence of anti-PLA $\mathrm{R}_{2}$ antibodies ranges from $52 \%$ to $69 \%$ in membranous nephropathy as determined by standardized methods. A recent study in a Chinese population showed that $82 \%$ of PMN patients had detectable anti-PLA $R$ antibody using an in-house Western blot assay. Anti-PLA $R$ antibodies are considered to be a serological marker in diagnosis [5], monitoring of disease activity [6], predicting prognosis [7], and response to immunosuppressive treatment [8] for PMN patients. However, previous studies always used only 1 in-house method to detect anti-PLA $R$ antibody. Given the important role of anti-PLA $R$ antibody as a clinical marker, a test system with high sensitivity and specificity is necessary. A recombinant cell-based indirect immunofluorescence assay that uses HEK293 cells overexpressing full-length PLA $_{2} R$ isoform 1 as substrate was recently developed to detect anti-PLA $R$ antibodies [9]. Moreover, an ELISA protocol with the PLA $R$ isoform 1 coated on the solid phase was also reported [10]. From the clinical perspective, the manifestation and disease status of patients are diverse and complicated, so it is often hard to stratify patients. Thus, it is practical and important to derive valuable information from a point-of-time test of hallmark biomarkers to guide the diagnostic pathway or therapy regimen.

Here, we attempted to use the 2 standard methods in a crosssectional style to investigate the anti-PLA $R$ antibody prevalence, as well as the relationship between anti-PLA $R$ antibody and renal function parameters, in Chinese patients with biopsy-proven PMN. The concordance of these 2 methods for detection of anti-PLA $R$ antibodies was also evaluated.

\section{Material and Methods}

\section{Subjects}

The sera of 82 patients with biopsy-proven PMN were collected consecutively from January 2011 to December 2013 in Nanfang Hospital, Southern Medical University. In this group, renal biopsy was performed for each patient with membranous nephropathy; the sera and urinary samples were collected within 1 week from the date of renal biopsy for each patient. We excluded patients with underlying diseases (e.g., systemic lupus erythematosus, viral hepatitis, and tumors) that suggested secondary membranous nephropathy (SMN) or those MN patients complicated with conditions such as nephropathy and renal vasculitis. As controls, we recruited 22 patients with SMN, 40 non-MN patients with established glomerulonephritis, and 20 healthy volunteers. Anti-PLA 2 R antibody was detected using both RC-IFA and ELISA for all serum samples. The results for 24-hour proteinuria, serum albumin, blood urea nitrogen (BUN), serum creatine, and serum cystatin $C$ were available for patients with PMN and SMN. The study was approved by the Ethics Committee of Nanfang Hospital and all subjects gave informed consent.

\section{Detection of anti-PLA $R$ antibodies by indirect immunofluorescence assay}

A recombinant cell-based indirect immunofluorescence assay (RC-IFA, EUROIMMUN, Lübeck, Germany) was used to detect anti-PLA $\mathrm{A}_{2} \mathrm{R}$ antibodies. RC-IFA contained a BIOCHIP mosaic of HEK 293 cells overexpressing full-length $P L A_{2} R$ isoform 1 (Uniprot reference [PLA R HUMAN]) and mock-transfected HEK293 cells as negative control. Fluorescein isothiocyanate (FITC)-conjugated goat anti-human IgG antibody was used for detection of bounded IgG-antibodies. The test was performed following the instructions of the manufacturer. The initial serum dilution was $1: 10$. All slides were evaluated by 2 independent observers using a fluorescence microscope (EUROStar III Plus, EUROIMMUN, Lübeck, Germany). In case of difference of opinion about a result, a third observer decided. A result was regarded as positive when a specific cytoplasmic, partly cell membrane or cell nuclei fluorescence, occurred on the transfected cells at a dilution of 1:10 or higher. For the positive sera, the titer of anti-PLA $R$ antibodies was determined at the end-point dilution.

\section{Detection of anti-PLA 2 antibodies by ELISA}

The serum level of anti-PLA $\mathrm{A}_{2} \mathrm{R}$ antibodies was detected quantitatively by means of ELISA (EUROIMMUN, Lübeck, Germany). The microplate was coated with the PLA $R$ isoform 1 . The serum samples were diluted 1:101 in sample buffer. Positive reaction was defined as concentration of anti-PLA $R$ antibody 
Table 1. Concordance between RC-IFA and ELISA for the detection of anti-PLA $\mathrm{R}_{2}$ antibodies.

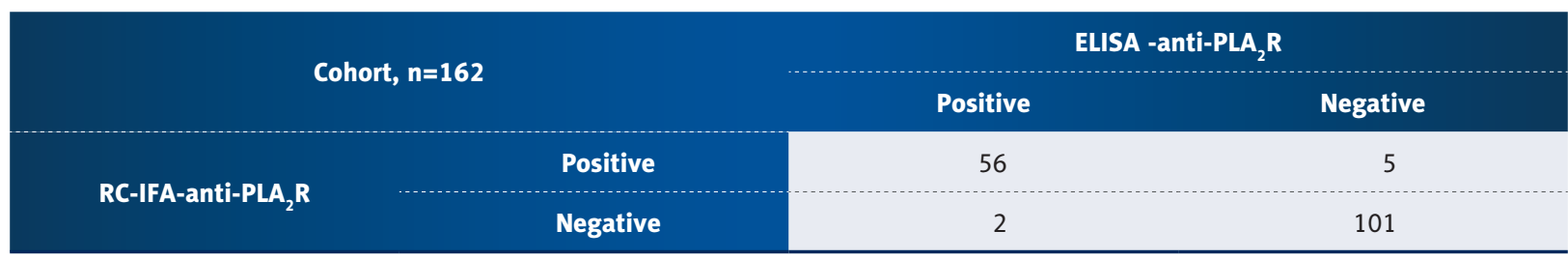

in serum $\geq 20 \mathrm{RU} / \mathrm{ml}$. If samples were outside the scale of the standard curve, samples were re-measured at a dilution of 1:400 or higher.

\section{Statistical analysis}

Data are presented as mean \pm standard deviation (SD) or median (25-75\% interquartile range). Student's t-test and KruskallWallis test were used for the comparison of different groups. For categorical variables, the chi-square test or Fisher's exact test was used to compare prevalence between groups, when applicable. Pearson's correlation coefficient (if data were normally distributed) or Spearman's rank correlation coefficient (if data were non-normally distributed) was used to analyze the correlation between parameters, including 24-hour proteinuria, serum albumin, blood urea nitrogen (BUN), serum creatine, serum cystatin $C$, and anti-PLA ${ }_{2} R$ antibody level.

All statistical analyses were performed by use of the SPSS 13.0 software package (SPSS Inc., Chicago, IL). $P<0.05$ was considered as statistically significant.

\section{Results}

\section{Demographic characteristics of the subjects}

The study cohort included 82 patients with PMN, 22 patients with SMN, 40 non-MN patients with established glomerulonephritis, and 20 healthy volunteers. SMN is often associated with lupus or hepatitis $B$ disease. Among the 22 patients with SMN, 15 were associated with hepatitis $B, 6$ with lupus, and 1 with both hepatitis $B$ and lupus. The non-MN group consisted of 22 cases with IgA nephropathy, 5 with crescent glomerulonephritis, 7 with focal segmental glomerular sclerosis, and 6 with membranoproliferative glomerulonephritis. Typically, the majority of patients with membranous nephropathy are middle-aged at the time of diagnosis. The sex ratio in PMN was close to 1: 1 (male/female: 42/40) and did not show sex bias for the Caucasian population [11]. In PMN, SMN, and non-MN cohorts, age was well matched $(47.44 \pm 16.15$ vs. $44.68 \pm 14.26$ vs. $48.28 \pm 10.94$ years).

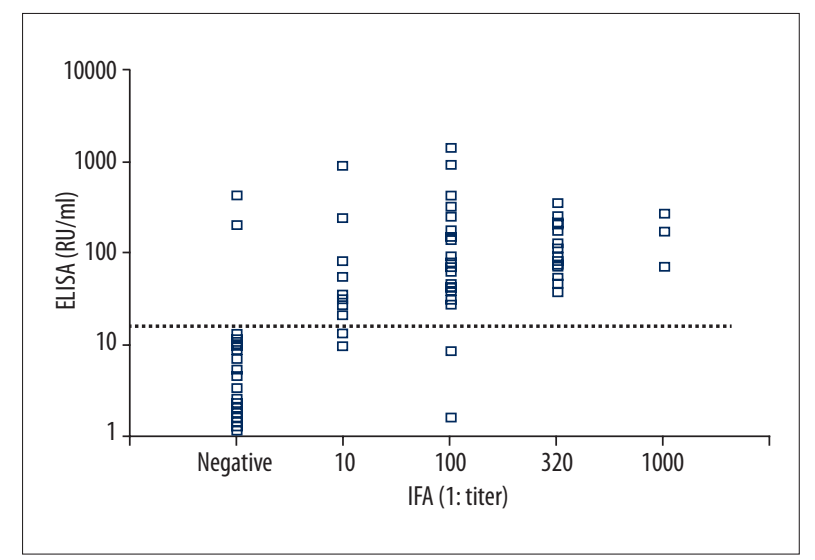

Figure 1. Significant correlation between anti-PLA $\mathrm{R}_{2}$ antibody levels detected by ELISA (RU/ml) and RC-IFA (1: titer) in PMN patients.

\section{Correlation of the 2 anti-PLA 2 antibodies test systems}

Of all the subjects enrolled in this study, the serum samples from 56 subjects were positive for anti-PLA $R$ antibodies in both RC-IFA and ELISA, while those from 101 subjects were negative in both assays. In 7 (4.3\%) MN patients, there was a discrepancy between both assays (Table 1). Two patients seronegative by RC-IFA were seropositive by ELISA. The 5 serum samples that were negative in the ELISA test generally gave low titers of $1: 10$ to $1: 100$ in the immunofluorescence test. Overall, the concordance rate between the 2 assays reached $95.7 \%$, with $\kappa=0.908$. In patients with $P M N$, anti-PLA ${ }_{2} R$ titers assessed by IFA and ELISA were further compared, and a good correlation between RC-IFA titer and ELISA results was obtained $(n=82, r=0.696, P<0.001)$ (Figure 1; the dashed line indicates the cut-off value $(20 \mathrm{RU} / \mathrm{ml})$ for anti-PLA ${ }_{2} \mathrm{R}$ antibodies detected by ELISA).

\section{Prevalence of anti-PLA ${ }_{2} R$ antibodies among groups}

Anti-PLA ${ }_{2} R$ antibodies were detectable in $64.6 \%$ (53/82) of PMN patients by RC-IFA. The antibody titers ranged from 1:10 to 1:1000. A similar prevalence was also found in PMN patients by means of ELISA (62.2\%, concentration range: $0-1423 \mathrm{RU} / \mathrm{ml}$ ). Both prevalences in PMN were significantly higher than those in the SMN group (RC-IFA: $64.6 \%$ vs. $36.4 \%$; ELISA: $62.2 \%$ vs. $31.8 \% ; P<0.05)$. Anti-PLA 2 R antibody was detectable only in 
Table 2. Prevalence of anti-PLA ${ }_{2} R$ antibodies detected by ELISA and RC-IFA among groups.

\begin{tabular}{|c|c|c|c|c|}
\hline & $\begin{array}{c}\text { PMN } \\
(n=82)\end{array}$ & $\underset{(n=22)}{S M N}$ & $\begin{array}{c}\text { Non-MN } \\
(n=40)\end{array}$ & $\begin{array}{l}\text { Health-Control } \\
\qquad(n=20)\end{array}$ \\
\hline RC-IFA-anti-PLA ${ }_{2} R$ & $53(64.6 \%)$ & $8(36.4 \%)^{a}$ & $0 \quad(0 \%)^{b}$ & $0 \quad(0 \%)^{b}$ \\
\hline ELISA-anti-PLA ${ }_{2} R$ & 51 (62.2\%) & $7(31.8 \%)^{\mathrm{a}}$ & $0 \quad(0 \%)^{b}$ & $0 \quad(0 \%)^{b}$ \\
\hline
\end{tabular}

a Compared with PMN, $P<0.05{ }^{\text {b }}$ compared with PMN, $P<0.001$. $P<0.05$ is considered statistically significant.

Table 3. Clinical characteristics in anti-PLA 2 R positive and negative patients with PMN.

\begin{tabular}{|c|c|c|c|c|c|c|}
\hline & \multicolumn{3}{|c|}{ RC-IFA } & \multicolumn{3}{|c|}{ ELISA } \\
\hline & Anti-PLA $\mathbf{A}_{2} \mathbf{R}(+)$ & Anti-PLA ${ }_{2} R(-)$ & $P$ value & Anti-PLA $\mathbf{A}_{2} \mathbf{( + )}$ & Anti-PLA $R(-)$ & $P$ value \\
\hline Age (years) & $45.77 \pm 15.65$ & $50.48 \pm 16.88$ & 0.209 & $46.55 \pm 15.76$ & $48.90 \pm 16.95$ & 0.526 \\
\hline Gender (male\%) & $27(50.9 \%)$ & $15(51.7 \%)$ & 0.946 & $27(52.9 \%)$ & $15(48.4 \%)$ & 0.689 \\
\hline Proteinuria (g/day)* & $5.80 \pm 4.91$ & $3.30 \pm 2.20$ & $0.016^{a}$ & $5.95 \pm 4.96$ & $3.23 \pm 2.14$ & $0.008^{b}$ \\
\hline Proteinuria $>3.5(\mathrm{~g} / \text { day })^{\mathrm{d}}$ & $30 \quad(60 \%)$ & $11(40.7 \%)$ & 0.164 & $30(62.5 \%)$ & $11(37.9 \%)$ & $0.036^{c}$ \\
\hline Serum albumin (g/dL) & $27.50 \pm 25.65$ & $26.89 \pm 8.89$ & 0.904 & $27.23 \pm 26.15$ & $27.37 \pm 8.70$ & 0.977 \\
\hline Blood urea nitrogen $(\mathrm{mg} / \mathrm{dL})$ & $6.48 \pm 5.07$ & $6.39 \pm 2.94$ & 0.929 & $6.56 \pm 5.15$ & $6.27 \pm 2.91$ & 0.773 \\
\hline Serum creatine (umol/L) & $71.25 \pm 27.87$ & $68.89 \pm 31.50$ & 0.728 & $71.94 \pm 28.33$ & $67.93 \pm 30.48$ & 0.549 \\
\hline
\end{tabular}

* The data of proteinuria is absent in 5 cases. ${ }^{a, b, c}$ Compared with anti-PLA $R$ negative group with RC-IFA or ELISA,P<0.05. ${ }^{\mathrm{d}}$ The data for this line was showed in form of number (\%) of patients whose proteinuria was above $3.5 \mathrm{~g} /$ day. $\mathrm{P}<0.05$ is considered statistically significanti.

PMN and SMN groups, while in the non-MN and healthy controls groups, there were no seropositive cases of anti-PLA $R_{2}$ antibody (Table 2). It also has comparable specificity, which was detected by RC-IFA and ELISA for the patients with PMN (RC-IFA: 90.2\%; ELISA: 91.5\%).

\section{Anti-PLA 2 reactivity correlates with proteinuria in PMN patients}

Proteinuria measurement was available in 77 (93.9\%) patients with PMN and 21 (95.5\%) patients with SMN. In 35 patients with PMN, proteinuria level was $<3.5 \mathrm{~g} /$ day at the time of blood sampling. Among these patients, 20 (57.1\%) were positive for anti-PLA ${ }_{2} R$ antibody and 13 (37.1\%) were negative as detected by both RC-IFA and ELISA. In all SMN patients with available proteinuria data, proteinuria level was $>3.5 \mathrm{~g} /$ day at the time of anti-PLA 2 antibody measurement.

As shown in Table 3, for PMN patients, proteinuria level was significantly higher in anti-PLA 2 R-positive patients than that in anti-PLA 2 R-negative patients when RC-IFA (anti-PLA2R positive vs. negative: $5.80 \pm 4.91$ vs. $3.30 \pm 2.20, P<0.05$ ) or ELISA (anti-PLA ${ }_{2} R$ positive vs. negative: $5.95 \pm 4.96$ vs. $3.23 \pm 2.14, P<0.05$ ) was used to detect anti-PLA $R$ antibodies. Furthermore, the proportion of cases with proteinuria level $\geq 3.5 \mathrm{~g} /$ day was significantly higher

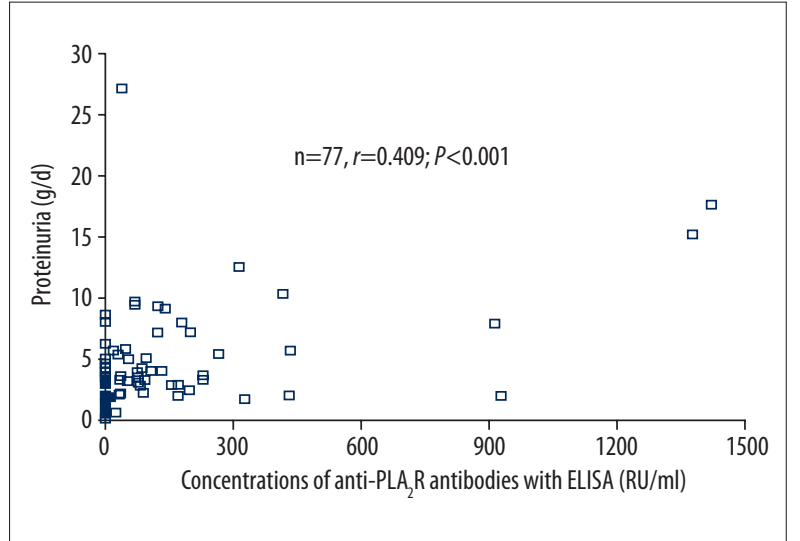

Figure 2. Significant correlation between concentration of anti-PLA $\mathrm{R}_{2}$ antibodies with ELISA and proteinuria in PMN. There is significant correlation $(n=77, r=0.409$, $P<0.001)$ between the anti-PLA 2 R level with ELISA and proteinuria in PMN. The data of proteinuria is absent in 5 cases, so only 77 data were included in analysis.

in anti-PLA ${ }_{2} \mathrm{R}$-ELISA-positive patients than in anti-PLA 2 R-ELISAnegative patients $(62.5 \%$ vs. $37.9 \%, P=0.036)$ (Table 3$)$. The overall correlation between the concentrations of anti-PLA ${ }_{2} R$ antibodies detected by ELISA and proteinuria was significant $(\mathrm{n}=77, r=0.409, P<0.001)$ (Figure 2). However, the levels of 
Table 4. Renal function parameters of patients with PMN based on graded concentrations of anti-PLA2R antibodies detected by ELISA.

\begin{tabular}{|c|c|c|c|c|}
\hline & \multicolumn{4}{|c|}{ Concentrations of anti-PLA2R antibodies } \\
\hline & Neg $(n=31)$ & 20-70 RU/ml (n=15) & 70-180 RU/mL (n=18) & $\geq 180 \mathrm{RU} / \mathrm{mL}(\mathrm{n}=18)$ \\
\hline Proteinuria (g/day) ${ }^{*}$ & $3.23 \pm 2.14$ & $5.63 \pm 6.64$ & $4.94 \pm 2.71$ & $7.26 \pm 5.33^{\mathrm{a}}$ \\
\hline Proteinuria >3.5 (g/day) & $11(37.9 \%)$ & $8(61.5 \%)$ & 11 (61.1\%) & 11 (64.7\%) \\
\hline Serum albumin (g/dL) & $27.37 \pm 8.70$ & $37.21 \pm 47.96$ & $23.33 \pm 7.32$ & $23.36 \pm 6.68$ \\
\hline Blood urea nitrogen $(\mathrm{mg} / \mathrm{dL})$ & $6.27 \pm 2.91$ & $8.49 \pm 8.67$ & $6.21 \pm 3.04$ & $5.43 \pm 2.25$ \\
\hline Serum creatine (umol/L) & $67.93 \pm 30.48$ & $74.86 \pm 29.71$ & $71.06 \pm 28.76$ & $70.56 \pm 28.32$ \\
\hline Serum cystatin C (mg/L) & $1.18 \pm 0.61$ & $1.15 \pm 0.50$ & $1.06 \pm 0.42$ & $1.19 \pm 0.62$ \\
\hline
\end{tabular}

${ }^{*}$ The data of proteinuria is absent in 5 cases. ${ }^{a}$ Compared with anti-PLA2R neg. group, $P<0.05 . P<0.05$ is considered statistically significant.

anti-PLA $\mathrm{R}_{2}$ antibody seemed to have no correlation with serum albumin ( $n=80, r=-0.072, P=0.526)$, blood urea nitrogen $(\mathrm{n}=81, r=-0.109, P=0.335)$, serum creatine $(n=81, r=0.006$, $P=0.955)$, and serum cystatin $C(n=82, r=-0.001, P=0.995)$ (data not shown). Anti-PLA 2 R-ELISA-positive cases were further divided into 3 groups based on the concentration of antibody. Antibody level $\geq 180 \mathrm{RU} / \mathrm{ml}$ was significantly correlated with higher proteinuria level $(P<0.05)$ (Table 4).

\section{Discussion}

In this study we investigated the performance of 2 detection methods, RC-IFA and ELISA, for anti-PLA $\mathrm{R}_{2}$ antibody and the correlation of anti-PLA $R$ antibody level with renal function parameters in Chinese patients with PMN. Our data show that anti-PLA $R$ antibody may be involved in the pathogenesis of PMN because the concentration of antibody was correlated with proteinuria, especially in patients with higher levels of antibody.

In the present study, RC-IFA and ELISA showed high overall consistency with each other, the same as the previous finding (94\% agreement, $\kappa=0.85$ ) [12]. Serum samples that were negative in the Anti-PLA 2 R ELISA had low titers, varying from 1:10 to $1: 100$, in the indirect immunofluorescence test. All sera with antibody titer of over 1:100 were also positive in the ELISA. Moreover, in terms of semi-quantitation or quantitation of anti-PLA $A_{2} R$, the titers of anti-PLA 2 determined by RC-IFA in the patient with $P M N$ correlated significantly with the concentrations of anti-PLA ${ }_{2} R$ by ELISA ( $\left.n=82, r=0.696, P<0.001\right)$, which was slightly lower than in a previous study [10]. RC-IFA and ELISA also showed similar specificity, which characterizes anti-PLA 2 R as the serological hallmark for PMN [13]. On the other hand, it was indicated in the present study that RC-IFA was more sensitive than ELISA, which was consistent with results reported previously [10]. This could be explained by the fact that in RC-IFA, antigen is expressed on the cell membrane with a natural conformation, while in ELISA, the epitope may adopt an unnatural conformation when it was adsorbed to the plate surface.

The positive rate of anti-PLA 2 antibodies detected by RC-IFA in the present study was $64.6 \%$ in PMN patients, which is higher than the $52 \%$ reported by Hoxha et al. [9], who first used RC-IFA for PMN. It was also higher than the rate reported in a recent study by Behnert et al. [14]. This positive prevalence was slightly lower than the $69 \%$ reported by Hofstra et al. and Svobodova et al. $[15,16]$, and consistent with the $63.7 \%$ demonstrated by Behnert et al. [17]. A recent study in Chinese patients reported that $82 \%$ of patients with PMN were anti$\mathrm{PLA}_{2} \mathrm{R}$-positive by using an in-house Western blot assay [18]. For anti-PLA 2 R antibody detected by ELISA in patients with $P M N$, the prevalence of $62.2 \%$ we obtained is lower than the $72 \%$ reported in European patients [12]. These differences may be attributed to differences in ethnicity, inclusion criteria, and assay technique. In our cohorts there were 29 patients with PMN without detectable PLA $R$ antibody in the serum, perhaps because the serum antibody had decreased due to the immunosuppressive therapy given before blood sampling. Having different antigens other than PLA $R$ may also be an explanation. Moreover, some patients might have been misclassified as having PMN when they actually had a secondary form of MN.

Anti-PLA ${ }_{2} \mathrm{R}$ antibodies were also present in SMN patients, with prevalence of more than $30 \%$ in our study, which is in contrast to the opinion that anti-PLA $R$ is generally absent in this cohort $[1,19]$. Nevertheless, Ronco et al. demonstrated that in some forms of secondary MN, the prevalence of anti-PLA $R$ antibodies range from $10 \%$ to $30 \%$ [20]. The prevalence in cancerrelated SMN was up to $28 \%$, which is higher than that in HBV infection-related SMN. Consistent with this report, Qin et al. used Western blot to detect anti-PLA 2 R and found that the autoantibodies were present in lupus MN, HBV-associated MN, and tumor-associated MN of Chinese patients [18]. Our study 
also found that SMN patients who had detectable antibody against PLA $R$ in serum, either by RC-IFA or ELISA, were all associated with HBV. SMN patients with HBV may be in an abnormal immune state during therapy, especially when receiving certain antiviral drugs. Therefore, the presence of anti-PLA $R$ in SMN could be dependent on the underlying diseases under investigation or the ethnic group involved.

In terms of the relationship between anti-PLA 2 reactivity and renal function parameters, the present data show that there were significant differences of the level of proteinuria and nephritic-range proteinuria ( $>3.5 \mathrm{~g} /$ day) between anti-PLA ${ }_{2} \mathrm{R}$ antibody-positive and -negative patients with PMN. Anti-PLA $R$ correlated significantly with proteinuria in PMN. This result confirms most findings from previous research $[6,12,21]$. The concentration of anti-PLA ${ }_{2} R$ detected by ELISA was significantly correlated with proteinuria (Figure 2). Anti-PLA $\mathrm{R}_{2}$ antibody concentration detected by ELISA had a significant effect on the level of proteinuria. The proteinuria level of patients with positive serum antibody level above $180 \mathrm{RU} / \mathrm{ml}$ was significantly higher than in those with negative antibody. Thus, as regards the correlation of anti-PLA $R$ with proteinuria, ELISA-anti-PLA $A_{2} R$ could be used to monitor the severity of PMN.

Many studies investigating the relationship between levels of anti-PLA $\mathrm{R}_{2}$ and proteinuria/disease activity had a prospective design in order to assess the monitoring utility of the autoantibodies, which needs a series of longitudinal test data obtained from 1 subject. In the present study, we investigated the correlation in a cross-sectional way without considering the heterogeneity of each subject (e.g., disease course or

\section{References:}

1. Beck LH Jr., Bonegio RG, Lambeau G et al: M-type phospholipase A2 receptor as target antigen in idiopathic membranous nephropathy. N Engl J Med, 2009; 361(1): 11-21

2. Kerjaschki D: Pathomechanisms and molecular basis of membranous glomerulopathy. Lancet, 2004; 364(9441): 1194-96

3. Salant DJ, Belok S, Madaio MP, Couser WG: A new role for complement in experimental membranous nephropathy in rats. J Clin Invest, 1980; 66(6): 1339-50

4. Stanescu HC, Arcos-Burgos M, Medlar A et al: Risk HLA-DOA1 and PLA(2)R1 alleles in idiopathic membranous nephropathy. N Engl J Med, 2011; 364(7): 616-26

5. Hu SL, Wang D, Gou WJ et al: Diagnostic value of phospholipase A2 receptor in idiopathic membranous nephropathy: a systematic review and meta-analysis. J Nephrol, 2014; 27(2): 111-16

6. Hofstra JM, Beck LH Jr., Beck DM et al: Anti-phospholipase A(2) receptor antibodies correlate with clinical status in idiopathic membranous nephropathy. Clin J Am Soc Nephrol, 2011; 6(6): 1286-91

7. Kanigicherla D, Gummadova J, Mckenzie EA et al: Anti-PLA $R$ antibodies measured by ELISA predict long-term outcome in a prevalent population of patients with idiopathic membranous nephropathy. Kidney Int, 2013; 83(5): 940-48

8. Beck LH Jr., Fervenza FC, Beck DM et al: Rituximab-induced depletion of anti-PLA $R$ autoantibodies predicts response in membranous nephropathy. Am Soc Nephrol, 2011; 22(8): 1543-50 biochemical status). In this setting, a strong correlation between the autoantibodies and proteinuria was observed. Therefore, a point-of-time test to determine levels of the autoantibodies probably reflected the disease severity, given that proteinuria can be used to identify patients at risk of rapid progression of chronic kidney disease [22]. It is well known that binding of circulating anti-PLA $R$ antibody to the PLA2R in the glomeruli leads to in situ formation of sub-epithelial deposits and a cascade of events, including proteinuria.

Our study has several limitations. Although this study enrolled the largest-ever Chinese population with PMN, the established correlation between the level of anti-PLA $\mathrm{A}_{2}$ antibody and proteinuria should be validated in a larger number of patients. Previous studies have demonstrated that anti-PLA 2 R antibody during the clinical course of patients with PMN was also correlated with remission or relapse. Because remission of proteinuria is the most meaningful indicator for good prognosis, we plan to observe in a Chinese population whether the correlation exists during follow-up.

\section{Conclusions}

In this study the prevalence of anti-PLA 2 R in PMN was about $62-64 \%$, which is an important serological marker for PMN diagnosis. RC-IFA and ELISA showed high agreement in detecting anti-PLA 2 R antibody in serum. Moreover, anti-PLA ${ }_{2} R$ detected by ELISA was significantly correlated with proteinuria in PMN patients, indicating that autoantibody against $P L A_{2} R$ could be used to assess the disease severity of PMN.

9. Hoxha E, Harendza S, Zahner G et al: An immunofluorescence test for phospholipase-A(2)-receptor antibodies and its clinical usefulness in patients with membranous glomerulonephritis. Nephrol Dial Transplant, 2011; 26(8): 2526-32

10. Dahnrich C, Komorowski L, Probst C et al: Development of a standardized ELISA for the determination of autoantibodies against human M-type phospholipase A2 receptor in primary membranous nephropathy. Clin Chim Acta, 2013; 421: 213-18

11. Schlumberger W, Hornig $N$, Lange $S$ et al: Differential diagnosis of membranous nephropathy with autoantibodies to phospholipase A2 receptor 1. Autoimmun Rev, 2014; 13(2): 108-13

12. Hofstra JM, Debiec H, Short CD et al: Antiphospholipase A2 receptor antibody titer and subclass in idiopathic membranous nephropathy. J Am Soc Nephrol, 2012; 23(10): 1735-43

13. Ronco P, Debiec H, Imai H: Circulating antipodocyte antibodies in membranous nephropathy: pathophysiologic and clinical relevance. Am J Kidney Dis, 2013; 62(1): 16-19

14. Behnert A, Fritzler MJ, Teng B et al: An anti-phospholipase A2 receptor quantitative immunoassay and epitope analysis in membranous nephropathy reveals different antigenic domains of the receptor. PLoS One, 2013; 8(4): e61669

15. Hoxha E, Kneissler U, Stege $G$ et al: Enhanced expression of the M-type phospholipase A2 receptor in glomeruli correlates with serum receptor antibodies in primary membranous nephropathy. Kidney Int, 2012; 82(7) 797-804 
16. Svobodova B, Honsova E, Ronco P et al: Kidney biopsy is a sensitive too for retrospective diagnosis of PLA R-related membranous nephropathy. Nephrol Dial Transplant, 2013; 28(7): 1839-44

17. Behnert A, Schiffer M, Muller-Deile J et al: Antiphospholipase A2 receptor autoantibodies: a comparison of three different immunoassays for the diagnosis of idiopathic membranous nephropathy. J Immunol Res, 2014; 2014: 143274

18. Qin W, Beck LH Jr, Zeng C et al: Anti-phospholipase A2 receptor antibody in membranous nephropathy. J Am Soc Nephrol, 2011; 22(6): 1137-43
19. Debiec $H$, Ronco P: PLA 2 R autoantibodies and PLA $A_{2} R$ glomerular deposits in membranous nephropathy. N Engl J Med, 2011; 364(7): 689-90

20. Ronco P, Debiec H: Pathogenesis of membranous nephropathy: recent advances and future challenges. Nat Rev Nephrol, 2012; 8(4): 203-13

21. Oh YJ, Yang SH, Kim DK et al: Autoantibodies against phospholipase A2 receptor in Korean patients with membranous nephropathy. PLoS One, 2013; 8(4): e62151

22. Iseki K: Chronic kidney disease: Proteinuria as a predictor of rapid eGFR decline. Nat Rev Nephrol, 2013; 9(10): 570-71 